\title{
Corrigendum to: Thermal inactivation kinetics of Aspergillus oryzae $\beta$-galactosidase in concentrated lactose solution
}

\author{
Viera Illeová, Milan Polakovič \\ Department of Chemical and Biochemical Engineering, Institute of Chemical and Environmental Engineering, \\ Faculty of Chemical and Food Technology, Slovak University of Technology, \\ Radlinského 9, 81237 Bratislava, Slovakia \\ milan.polakovic@stuba.sk
}

Corrigendum to: Acta Chimica Slovaca, 11(2): 170-174 (2018). DOI: 10.2478/acs-2018-0024

We would like to amend a mistake in our paper (Illeová and Polakovič, 2018) where wrong graphs

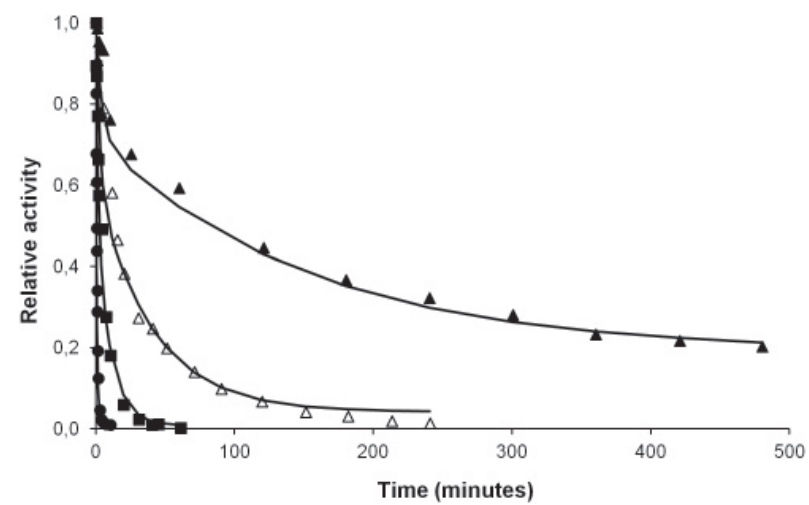

Fig. 1. Thermal inactivation of $\beta$-GAL in a reaction medium with the initial lactose concentration of $300 \mathrm{~g} / \mathrm{L}$. Symbols represent experimental data at individual temperatures: $-75^{\circ} \mathrm{C}, \boldsymbol{\square}-70^{\circ} \mathrm{C}$, $\triangle-67.5^{\circ} \mathrm{C}, \Delta-65^{\circ} \mathrm{C}$. Lines are the fitted activity values using the model specified by Eqs. (1)—(3).

\section{References}

Illeová V, Polakovič M (2018) Acta Chim. Slovaca 11(2): $170-174$. were assigned to Figures 1 and 2. Corrected figures and captions are provided below.

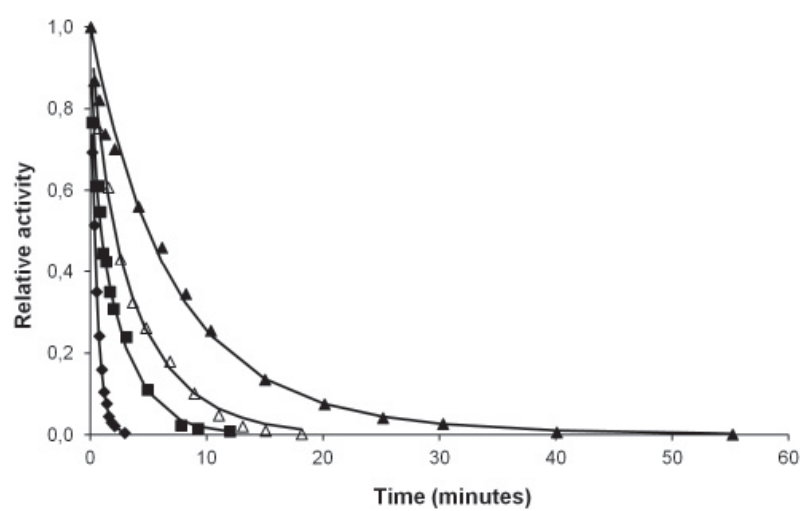

Fig. 2. Thermal inactivation of $\beta-G A L$ in lactosefree buffer. The meaning of all symbols and lines is the same as in Fig. 1 except for the experimental data at $72.5^{\circ} \mathrm{C}(\bullet)$. 